\title{
Guide to Abstract Numbers
}

\begin{abstract}
PLxx where xx indicates the number of the plenary lecture. Abstracts for symposiums are designated by the alphanumeric SPxx-n where xx indicates the topic of the symposium and $\mathrm{n}$ indicates the number of the presentation in that symposium. The symposium topics are listed below. The symposium topics are listed below. Abstracts for oral presentations are designated by the alphanumeric OPxx-n where $\mathrm{xx}$ indicates the topic of the presentation and $\mathrm{n}$ indicates the number of the presentation within that topic. The oral presentation topics are listed below. Abstracts for poster presentations are designated by the alphanumeric PPxxx where xxx indicates the number of the poster presentation. Abstracts for satellite symposiums are designated by the alphanumeric SSxx-n where $\mathrm{xx}$ indicates the topic of the symposium and $\mathrm{n}$ indicates the number of the presentation in that symposium. The oral presentation topics are listed below.
\end{abstract}

Symposiums Topics:

SP01 BPPV-Update \& Controversies

SP02 The Inner Ear Imaging

SP03 Vascular Vertigo

SP04 VEMP (Clinical) - Clinical Application of VEMPs

SP05 Meniere's Disease (Clinical) - Intratympanic Drug Application

SP06 Vestibular Migraine

SP07 Head Impulse Test

SP08 Gait \& Posture

SP09 Video Head Impulse Test (Round Table)

SP10 Basic Physiology of Vestibular Compensation and Adaptation

SP11 The 3rd Joint Meeting of The Korean Balance Society and Japan Society for Equilibrium Research

SP12 Vestibular Rehabilitation

SP13 Vestibular Prosthesis

SP14 Neurosensory Diagnosis and Prognosis of Mild Traumatic Brain Injury

SP15 Genetics for Inner Ear/Vestibular Disorder

SP16 Superior Canal Dehiscence Syndrome

SP17 Vestibular Brain and Functional Imaging

SP18 Spatial Orientation

SP19 Meniere's Disease (Basic) - Recent Progress in Revealing Pathologic Mechanism of Meniere's Disease

SP20 Visually Induced Dizziness; What Is It; Where Does It Come From; How Can We Deal With It?

SP21 Vestibular Disorder and Autonomic Interaction

SP22 BPPV-Pathogenesis

SP23 Cochlear Implant and Vestibular Function

SP24 Pathophysiologic Mechanism of Functional and Psychiatric Vestibular Disorders

SP25 VEMP (Basic) - The Neural Basis of Vestibular Evoked Myogenic Potentials

Oral Presentations Topics:

OP01 VEMP and Otolithic Function Tests I

OP02 Vestibular Migraine

OP03 Epidemiology

OP04 Meniere's Disease and Related Disorders I 
OP05 Central Vestibular Disorder I

OP06 Perilymphatic Fistula and Superior Canal Dehiscence Syndrome OP07 Benign Paroxysmal Position Vertigo I

OP08 Head Impulse Test I

OP09 Clinical Tests for Vestibular Function I

OP10 Ocular Motility: Physiology and Pathology

OP11 Functional, Psychiatric, Autonomic Disorders and Others I

OP12 Animal Models and Molecular Approach

OP13 Meniere's Disease and Related Disorders II

OP14 Bilateral Vestibulopathy and Vestibular Prosthesis

OP15 Meniere's Disease and Related Disorders III

OP16 Vestibular Neuritis

OP17 Anatomy and Physiology

OP18 Clinical Tests for Vestibular Function II

OP19 Gait, Posture, and Spatial Orientation I

OP20 Cochlear Implantation and Vestibular Function

OP21 Benign Paroxysmal Position Vertigo II

OP22 VEMP and Otolithic Function Tests II

OP23 Vestibular Compensation and Rehabilitation I

OP24 Vascular Vertigo

OP25 Functional, Psychiatric, Autonomic Disorders and Others II

OP26 Head Impulse Test II

OP27 Gait, Posture, and Spatial Orientation II

OP28 Vestibular Compensation and Rehabilitation II

OP29 Imaging of the Inner Ear and Vestibular System

OP30 Central Vestibular Disorder II

Satellite Symposiums Topics:

SS01 Neural Coding and Multimodal Interactions for Action and Perception

SS02 Vestibular Periphery: Structure, Function and Restoration

SS03 Vestibular Plasticity and Adaptation

SS04 Neurophysiology of Vestibular Disorders and Treatments 